\title{
Occurrence and distribution of Giardia species in wild rodents in Germany
}

\author{
Yosra A. Helmy ${ }^{1,4,5}$, Nastasja G. Spierling ${ }^{2}$, Sabrina Schmidt ${ }^{2,6}$, Ulrike M. Rosenfeld ${ }^{2}$, Daniela Reil ${ }^{3}$, Christian Imholt ${ }^{3}$,
} Jens Jacob ${ }^{3}$, Rainer G. Ulrich², Toni Aebischer ${ }^{1}$ and Christian Klotz ${ }^{1 *}$

\begin{abstract}
Background: Giardiasis is an important gastrointestinal parasitic disease in humans and other mammals caused by the protozoan Giardia duodenalis. This species complex is represented by genetically distinct groups (assemblages A-H) with varying zoonotic potential and host preferences. Wild rodents can harbor potentially zoonotic assemblages A and B, and the rodent-specific assemblage G. Other Giardia spp. found in these animals are Giardia muris and Giardia microti. For the latter, only limited information on genetic typing is available. It has been speculated that wild rodents might represent an important reservoir for parasites causing human giardiasis. The aim of this study was to investigate the occurrence and distribution of Giardia spp. and assemblage types in wild rodents from different study sites in Germany.

Results: Screening of 577 wild rodents of the genera Apodemus, Microtus and Myodes, sampled at eleven study sites in Germany, revealed a high overall Giardia prevalence. Giardia species determination at the SSU rDNA gene locus revealed that Apodemus mice, depending on species, were predominantly infected with one of two distinct G. muris sequence types. Giardia microti was the predominant parasite species found in voles of the genera Microtus and Myodes. Only a few animals were positive for potentially zoonotic G. duodenalis. Subtyping at the betagiardin (bg) and glutamine dehydrogenase ( $g d h)$ genes strongly supported the existence of different phylogenetic subgroups of $G$. microti that are preferentially harbored by distinct host species.

Conclusions: The present study highlights the preference of G. muris for Apodemus, and G. microti for Microtus and Myodes hosts and argues for a very low prevalence of zoonotic $G$. duodenalis assemblages in wild rodents in Germany. It also provides evidence that G. muris and G. microti subdivide into several phylogenetically distinguishable subgroups, each of which appears to be preferentially harbored by species of a particular rodent host genus. Finally, the study expands the database of sequences relevant for sequence typing of $G$. muris and $G$. microti isolates which will greatly help future analyses of these parasites' population structure.
\end{abstract}

Keywords: Giardia spp., Protozoan infection, Sequence typing, Wild rodents, Reservoir

\section{Background}

Giardiasis caused by the flagellated protozoan Giardia duodenalis (syn. G. lamblia and G. intestinalis) is one of the most frequent gastrointestinal parasitic diseases in humans worldwide [1, 2]. Giardia duodenalis is considered as a species complex of genetically different but morphologically identical organisms with varying zoonotic potential and host preferences. Humans and a wide range of mammal species including livestock, pets and wildlife

\footnotetext{
* Correspondence: klotzc@rki.de

${ }^{1}$ Unit 16 Mycotic and Parasitic Agents and Mycobacteria, Department of

Infectious Diseases, Robert Koch-Institute, 13353 Berlin, Germany

Full list of author information is available at the end of the article
}

are susceptible to these parasites [3]. This species complex consists of at least eight genetically distinct groups (referred to as assemblages $\mathrm{A}$ to $\mathrm{H}$ ) based on sequence analyses of a few genomic markers such as glutamate dehydrogenase $(g d h)$, beta-giardin $(b g)$ and small-subunit rRNA (SSU rDNA) genes [4-9]. It has been suggested to consider part of these assemblages as distinct Giardia species due to their genetic distance and differing host preferences [3]. Giardia duodenalis assemblages A and B show the broadest host range, which includes humans and, therefore, they are considered potentially zoonotic $[3,8,10]$. Assemblages $\mathrm{C}-\mathrm{H}$ are mainly found in certain mammal taxa: $\mathrm{C}$ and $\mathrm{D}$ in canids, $\mathrm{E}$ in ruminants, $\mathrm{F}$ in 
felids, $\mathrm{G}$ in rodents, and $\mathrm{H}$ in marine mammals $[3,11]$. The overall impact of zoonotic transmission on the epidemiology of human infections remains unclear [3].

Besides G. duodenalis, to date two more Giardia species, Giardia microti and Giardia muris, are known to infect mammals. Both species are found in small rodents with supposedly different host preferences. Giardia microti is thought to be a parasite mainly of rodents belonging to the family Cricetidae such as voles and muskrats and G. muris mainly of rodents belonging to the family Muridae [3,12-15]. Thus, wild rodents can be infected with different Giardia species, including $G$. microti, G. muris and various assemblages of G. duodenalis (assemblages A, B and G) [3, 15-18]. To evaluate the epidemiology and the potential zoonotic risk, molecular surveys for Giardia spp. in various wild rodents and lagomorphs, including the North American beaver (Castor canadensis), muskrat (Ondatra zibethicus), brown rat (Rattus norvegicus), house mouse (Mus musculus) and prairie vole (Microtus ochrogaster), have been attempted to determine the Giardia species or assemblage types. However, systematic molecular studies are still comparatively rare, in particular if one considers the broad range of existing rodent host species $[8,9,13,15,17-20]$. In fact, earlier studies suggested a link of waterborne human giardiasis outbreaks to a source in wildlife, in particular beavers, that led to the classification of Giardia as a zoonotic agent $[21,22]$. However, the distribution of different Giardia species in rodents of various genera and their geographical distribution based on molecular studies is not sufficiently clarified [21].

Rodents can carry a multitude of pathogens, including important zoonotic viruses, bacteria and parasites [2325 ] and as infections with Giardia spp. are common in wild rodents it has been discussed whether they could play an important role as a reservoir for these potentially zoonotic parasites as well [17, 19, 24, 26].

The goal of this study was to investigate the occurrence and distribution of Giardia spp., including Giardia species allocation, in wild rodents from different study sites in Germany.

\section{Results}

\section{Frequent occurrence of Giardia spp. in wild rodents in Germany}

Fecal samples of 577 small mammals, collected between 2011 and 2012, were included in the analysis. Samples were collected at eleven sampling sites across four federal states of Germany (Additional file 1: Figure S1, Table 1). Sampling sites fell into five distinct geographical regions (Additional file 1: Figure S1). Sampled animals belonged to six species of three rodent genera: Apodemus $[n=93,16 \%$; including Apodemus agrarius (striped field mouse), Apodemus flavicollis (yellow- necked mouse) and Apodemus sylvaticus (wood mouse)]; Microtus [ $n=181,31 \%$; including Microtus agrestis (field vole) and Microtus arvalis (common vole)]; and Myodes [ $n=303,52 \%$; represented by Myodes glareolus (bank vole)]; see Table 1 for further details and local distribution.

All samples were initially tested by immunofluorescence assay (IFA) and cyst-like particles reacting with cyst-wall specific antibodies were found in 423 of 577 samples (73\%, 95\% confidence interval, 95\% CI: $70-77 \%)$. The prevalence as revealed by the IFA analysis was significantly different between the genera Microtus (159 of 181 samples, 88\%, 95\% CI: 82-92\%), Myodes (239 of 303 samples, 79\%, 95\% CI: 74-83\%) and Apodemus (25 of 93 samples, 27\%, 95\% CI; 18-37\%) (adjusted Fisher's exact test P-values: Microtus vs Myodes $P=0.014$; Microtus vs Apodemus $P<0.0001 ;$ Myodes vs Apodemus $P<0.0001$ ). Differences among species of the same genus or among the same species from different study sites were not observed or could not be tested appropriately due to low sample size (Table 1, Additional file 2: Table S1).

Subsequently DNA was extracted and analyzed by previously described real time-PCR (qPCR) [27] and seminested PCR assays (SSU-PCR) $[4,28]$ targeting the SSU rDNA gene locus. The respective number of samples yielding specific PCR products was 471 of 569 (83\%, 95\% CI: $79-86 \%)$ for the qPCR and 371 of $569(65 \%$, 95\% CI: 61-69\%) for the SSU-PCR assay. Of note, in all three rodent genera a higher number of infected animals were detected by qPCR compared to IFA analyses, possibly due to higher sensitivity of the former assay as previously suggested [27, 29] (Table 1). The qPCR and $S S U$-PCR tests overall confirmed the higher Giardia prevalence in Microtus and Myodes compared to Apodemus (Table 1 and data not shown).

Since samples of Apodemus spp. were less frequently positive than those of Microtus and Myodes, we estimated parasite abundance to test whether a lower abundance could in part explain this finding. For this purpose we performed a semi-quantitative analysis of the cyst numbers in fecal samples using the IFA data and of parasite DNA abundance in feces using the threshold cycle $(\mathrm{Ct})$ value of the $\mathrm{qPCR}$ results. The latter served as a proxy for parasite numbers. DNA samples of Apodemus feces showed significantly higher Ct-values when amplifying Giardia SSU rDNA compared to samples from Myodes (delta Ct $\sim 2 ; P<0.001$ ) and Microtus (delta $\mathrm{Ct} \sim 4 ; P<0.001$ ) (Table 2). This implies on average 4-16 times less Giardia DNA content in Apodemus samples compared to those of Myodes and Microtus (Table 2) and, most likely, reflects the lower abundance of parasite cysts in Apodemus animals confirmed by semi-quantitative analysis of IFA data (Table 2). The qPCR Ct-values for Myodes and Microtus were also 


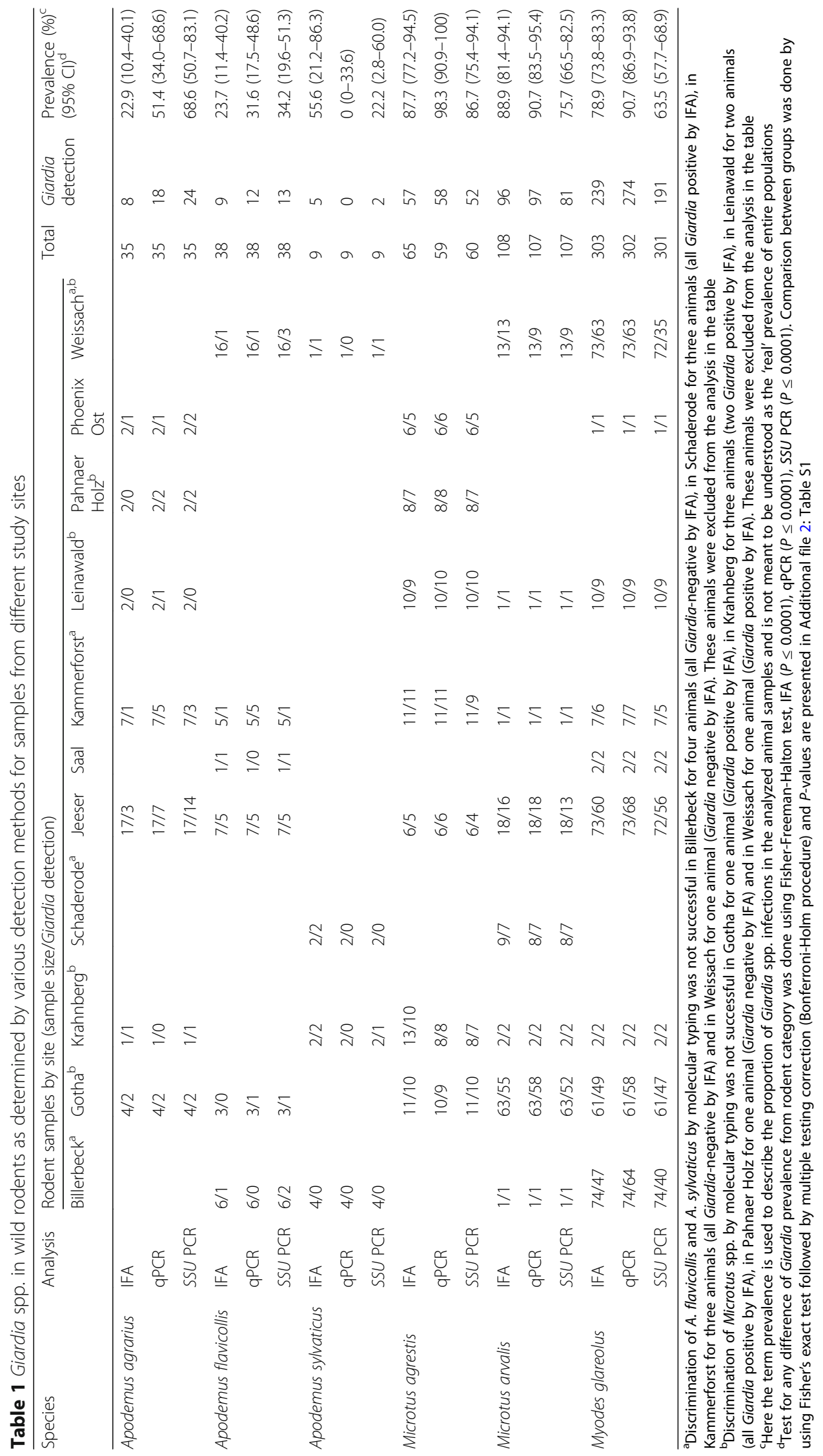


Table 2 Relative Giardia abundance in rodent samples. Relative Giardia abundance in rodent samples was determined by semi-quantitative IFA and by analysis of ct-values of Giardia positive samples in a Giardia specific qPCR assay ${ }^{\mathrm{a}}$

\begin{tabular}{|c|c|c|c|c|c|c|c|c|}
\hline \multirow[t]{2}{*}{ Genus } & \multirow[t]{2}{*}{ Species } & \multicolumn{3}{|l|}{ Ct values (qPCR) } & \multicolumn{4}{|c|}{ Semi-quantitative cyst abundance (IFA) $)^{c}$ absolute numbers (\%) $[95 \% \mathrm{Cl}]$} \\
\hline & & Median (Range) ${ }^{b}$ & $95 \% \mathrm{Cl}$ & $n$ & + & ++ & +++ & $n$ \\
\hline \multirow[t]{3}{*}{ Apodemus } & agrarius & $33.9(23.5-38.1)$ & $32.2-35.4$ & 18 & $8(100)[63.1-100]$ & $0(0)[0-36.9]$ & $0(0)[0-36.9]$ & 8 \\
\hline & flavicollis & $32.9(23.9-39.9)$ & $30.7-35.6$ & 12 & $6(86)[42.1-99.6]$ & $1(14)[0.4-57.9]$ & $0(0)[0-40.0]$ & 7 \\
\hline & sylvaticus & no ct & & & $5(100)[47.8-100]$ & $0(0)[0-52.2]$ & $0(0)[0-52.2]$ & 5 \\
\hline Total $^{d}$ & & $33.4(23.5-39.9)$ & $32.4-34.6$ & 34 & 22 (96) [78.1-99.8] & 1 (4) [0-21.9] & $0(0)[0-14.8]$ & 23 \\
\hline \multirow[t]{2}{*}{ Microtus } & agrestis & $28.1(22.9-36.3)$ & $27.5-29.1$ & 58 & $43(80)$ [66.5-89.4] & 10 (19) [9.3-31.4] & $1(2)[0.1-12.3]$ & 54 \\
\hline & arvalis & $29.0(20.9-38.3)$ & $28.4-30.0$ & 97 & $57(58)[47.2-67.5]$ & 28 (28) [19.7-38.2] & 14 (14) [8.0-22.6] & 99 \\
\hline Total $^{d}$ & & $28.5(20.9-38.3)$ & $28.4-29.5$ & 163 & $104(70)$ [62.7-77.9] & 25 (17) [11.3-24.1] & 18 (12) [7.4-18.6] & 147 \\
\hline Myodes & glareolus & $30.7(22.8-39.9)$ & $30.4-31.2$ & 274 & 149 (64) [57.7-70.4] & 50 (21) [16.4-27.4] & 33 (14) [10.0-19.4] & 232 \\
\hline
\end{tabular}

Abbreviations: $C t$ threshold cycle, IFA immunofluorescence assay, $n$ sample size, $q P C R$ real-time PCR, $95 \%$ Cl $95 \%$ confidence interval

${ }^{a}$ One should note that parasite excretion is often not uniform. However, it is assumed that such effects averaged out by analyzing the means of different groups ${ }^{b}$ Significant differences of median-values analyzed by non-parametric Kruskal-Wallis test $(\mathrm{H}=53.8, P<0.0001)$. Post-hoc test, Dunn's test of multiple comparisons using rank sums: Ct-values were different between genera (Apodemus vs Microtus $P<0.0001$, Apodemus vs Myodes $P<0.0001$, Microtus vs Myodes $P<0.0001$; mean ranks for Apodemus $=353.9$, for Microtus=183.5 and for Myodes=252.6). There were no significant differences within the species of the same genus

${ }^{c}$ Fecal samples were distributed on slides using inoculation loop $(\sim 10 \mu \mathrm{l})$ and analyzed by IFA. Cyst amount of the samples were semi-quantified (1-10 cysts ( +$)$, 11-50 cysts $(++),>50$ cysts $(+++)$. Comparison of $(+)$ vs $(++/+++)$ between groups was done using Fisher's exact test followed by multiple testing correction (Bonferroni-Holm procedure): Apodemus vs Microtus $(P=0.019)$; Apodemus vs Myodes $(P=0.005)$; Microtus vs Myodes $(P=0.218)$

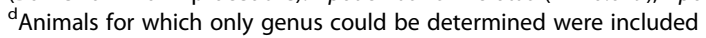

significantly different, but such a difference was not noted at the resolution of the semi-quantitative IFA test (Table 2). Thus, Giardia burden is likely to differ among rodents of different genera and decreasing when comparing Microtus and Myodes vs Apodemus.

\section{Giardia muris and G. microti predominate in wild rodents while zoonotic Giardia spp. were rarely detected}

To determine the Giardia species in wild rodents the sequences of 371 (of $n=571$ investigated) PCR products of the semi-nested SSU PCR (see above) were determined, analyzed and compared to reference sequences (Table 3). Overall, 358 sequences could be typed and 355 of these sequences could be assigned to Giardia spp. and three sequences to Octomitus intestinalis, a sister lineage of Giardia spp. [30].

The analysis revealed that $97 \%$ of Microtus and $96 \%$ of Myodes were infected with G. microti. Apodemus spp. were predominantly infected with $G$. muris $(80 \%)$ but a sizeable fraction contained G. microti (18\%) (see Table 3 for details). There were apparent differences in the $G$. muris/G. microti proportion between $A$. agrarius and $A$. flavicollis, but absolute numbers of samples from these two species were too low to allow meaningful conclusions (Table 3).

Only five samples (of $n=358,1.4 \%$; Table 3 ) were found positive for G. duodenalis, and respective sequences could be assigned to assemblage A (in case of one Apodemus, two Myodes samples) and B (in two samples from Myodes). Attempts to further subtype these samples at different genomic loci (tpi, $b g$ and $g d h)$ were not successful (data not shown).
Of the three $O$. intestinalis-positive samples (two from Microtus, one from Myodes) two were positive in the IFA analysis and all samples were also positive in the Giardia-specific qPCR analysis. Further subtyping at $b g$ locus (see below) from the two IFA positive samples revealed G. microti as a result indicating possible $G$. microti/O. intestinalis co-infections in these animals.

\section{Sequence analysis of SSU rDNA, $b g$ and $g d h$ genes revealed high variation in G. microti and G. muris} Sequence analysis was performed first on 317 SSU rDNA sequences (277 G. microti, 5 G. duodenalis, 32 G. muris and $3 \mathrm{O}$. intestinalis) for which a complete sequence from both amplicon DNA strands was available (see Additional file 3: Figure S2 for dendrogram of Bayesian phylogenetic analysis of all SSU rDNA samples, including corresponding data of host species, locality, season, habitat and year of collection). Unique sequences were deduced from this data set and analyzed for possible phylogenetic relatedness which indicated high divergence within G. muris and G. microti sequences (Fig. 1). This prompted us to also type samples at the $g d h$ and $b g$ gene loci by established methods [5, 7] (Fig. 2).

Within the 32 G. muris SSU rDNA sequences, 10 unique sequence types were identified with four types represented by $\geq 3$ identical sequences. Distance analysis revealed that 31 sequences differed by $0-10$ single nucleotide polymorphisms (SNPs) to each other over a fragment length of 245-247 base pairs (bp). In contrast, one sample (ssu406, from M. glareolus) differed by 19-26 SNPs to these 31 sequences and this sequence was identical to the G. muris sequence AF113895 that was used for reference. The separation of the 31 


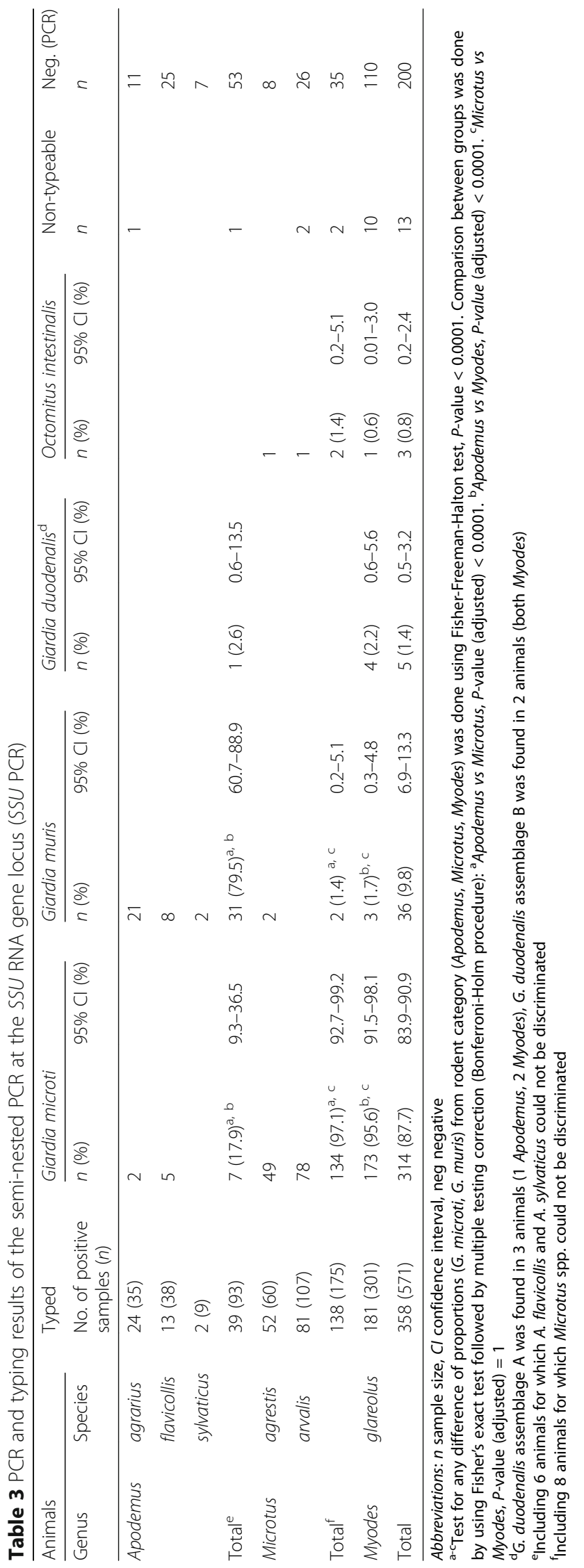




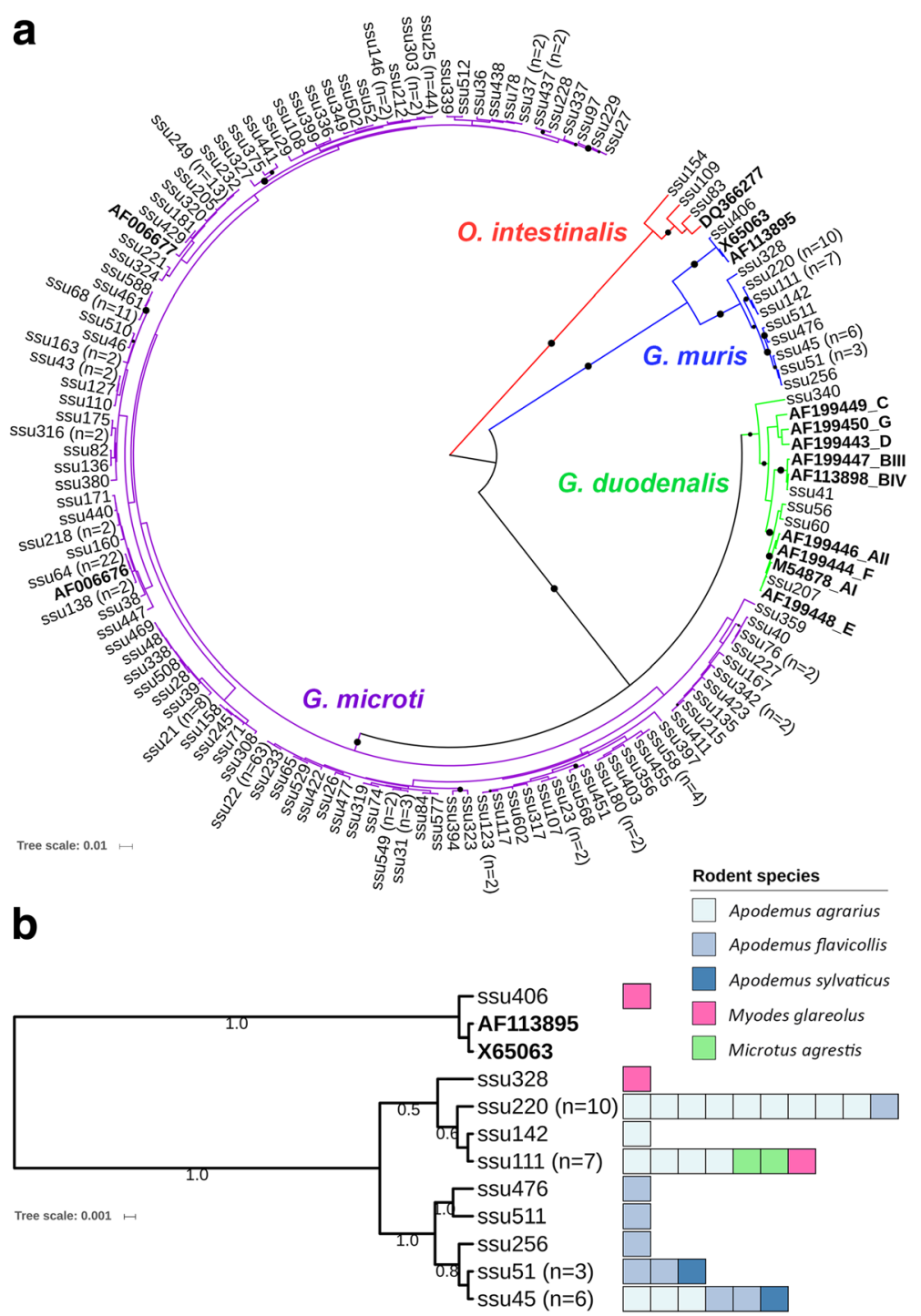

Fig. 1 Bayesian phylogenetic analysis of unique SSU rDNA sequence fragments. Sequences of representative samples are shown and number of samples with identical sequences is given in brackets. Maximum likelihood analysis based on PhyML resulted in similar trees (not shown). a Unrooted phylogenetic tree comprised of 106 unique SSU rDNA sequences that have been classified as G. microti (purple clade), 10 unique sequences classified as G. muris (blue clade), 5 sequences classified as G. duodenalis (green clade) and 3 sequences classified as $O$. intestinalis (red clade), a sister lineage of Giardia spp. Reference sequences (GenBank accession numbers) of O. intestinalis, G. muris, G. duodenalis and G. microti are marked in bold. Posterior probabilities $\geq 0.5$ are illustrated by black dots (proportionally increasing in size). $\mathbf{b}$ Unrooted phylogenetic tree of the 10 unique G. muris sequences and 2 references (GenBank: AF113895, X65063). Only posterior probabilities $\geq 0.5$ are highlighted. Rodent species and number of samples from which unique sequences derived are illustrated in color bars (one square size represents one animal). Testing for significant phylogeny-trait correlations within the G. muris subgroup for host distribution clustering using the programme BaTS [51] revealed the following P-values: A. agrarius $\leq 0.001$, A. flavicollis $=0.079$

sequences deduced here from current G. muris GenBank entries AF113895 and X65063 was also strongly supported by phylogenetic analyses that revealed a separation into two newly identified sequence clusters. Each of these sequence clusters was preferentially detected in different Apodemus hosts as determined by using phylogenetic trait analysis (Fig. 1b). Analysis of $b g$ sequences identified five G. muris sequence types for this typing fragment. These sequences were also separated from $b g$ GenBank sequence EF455599 used as reference further supporting the existence of phylogenetically distinguishable subgroups within the $G$. muris population (Fig. 2). No gdh sequences could be amplified of G. muris DNA containing samples and, notably, also no database entry exists for this gene.

Giardia microti SSU rDNA differed by $0-17$ SNPs to each other over a fragment length of $250 \mathrm{bp}$. Within the G. microti samples, 106 unique sequences were identified, 


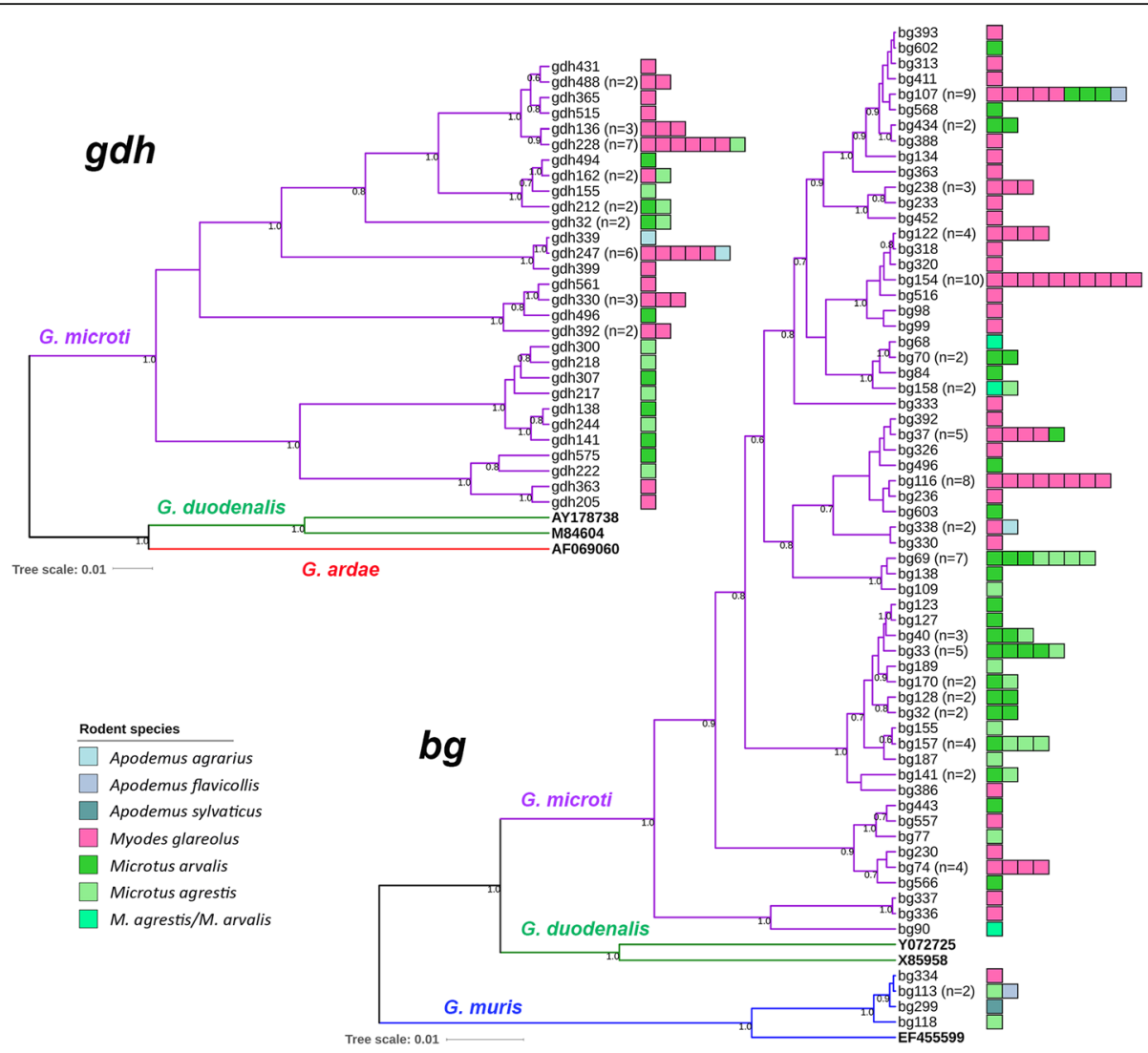

Fig. 2 Bayesian phylogenetic analysis of unique $g d h$ and $b g$ sequence fragments. Sequences of representative samples are shown and number of samples with identical sequences is given in brackets. Unrooted phylogenetic trees comprised of 29 unique gdh and 59 unique bg sequences that have been classified as G. microti (purple clade) and 4 unique bg (and none gdh) sequences classified as G. muris (blue clade). Further reference sequences (GenBank accession numbers) for bg were included: G. muris (EF355599) and G. duodenalis (X85958, assemblage Al; Y072725, assemblage B; green clade). References for gdh included G. duodenalis (M84604, assemblage Al; AY178738, assemblage B; green clade) and G. ardae gdh (AF069060; red clade) sequences. Reference sequences are marked in bold. Only posterior probabilities $\geq 0.5$ are highlighted. Rodent species and number of samples from which unique sequences derived are illustrated in color bars (one square size represents one animal). Maximum likelihood analysis based on PhyML resulted in similar trees (not shown). Testing for significant phylogeny-trait correlations within the G. microti subgroup for host distribution clustering using the programme BaTS [51] revealed the following P-values: gdh, Myodes $=0.079$, Microtus $\leq 0.001 ;$ bg, Myodes $\leq 0.001$, Microtus $\leq 0.001$

including 6 types represented by 8, 11, 13, 22, 44 and 63 identical sequences, respectively. The phylogenetic analysis is compatible with a genetic substructure of the population as previously suggested [13] although, expectedly, support of nodes was low due to the short sequence fragment used for the analysis (Fig. 1a). However, analysis of $29 \mathrm{G}$. microti gdh (unique sequence types deduced from a respective set of 49 total G. microti sequences) and 59 G. microti bg (deduced from a total set of 118 G. microti sequences of this locus) sequences strongly supports the existence of different phylogenetic subgroups in G. microti (Fig. 2). Testing for a significant association with host distribution (Microtus vs Myodes) of the phylogenetic subgroups using phylogenetic trait analysis indicated that subgroups are preferentially harbored by one of the two host genera (Fig. 2).

\section{Discussion}

Rodents investigated in this study belonged to mice of the genus Apodemus (A. agrarius, A. flavicollis or A. sylvaticus) or to voles of the genus Microtus (M. agrestis or $M$. arvalis) or Myodes (M. glareolus). The overall occurrence of Giardia spp. in these animals was very high. Apodemus spp. were mainly infected with $G$. muris, whereas G. microti was predominantly found in Microtus spp. and M. glareolus. Our data argue that G. muris and G. microti subdivide into several phylogenetically distinguishable subgroups, each of which appears to be preferentially harbored by species of a particular rodent host genus. Only a low proportion of samples (1.4\%) contained G. duodenalis assemblages A or B. This implies a very low potential risk for transmission of zoonotic Giardia types from wild rodents in Germany. 
In contrast, several previous studies estimated a higher potential risk for zoonotic transmission from Giardia-infected wild rodents $[17,19,24,26,31-34]$. However, in only a few of these reports molecular analysis was performed to identify the underlying Giardia species. These studies concerned very different geographical regions and covered a variety of different rodent species: for example, G. duodenalis assemblages $G$ and B in mice (Mus spp.) and rats (Rattus spp.) [17, 20], G. duodenalis assemblage B and G. microti in muskrats (O. zibethicus) $[9,13]$, and G. microti in prairie voles (M. ochrogaster) [13]. A study from Sweden found G. muris and G. duodenalis assemblage $\mathrm{G}$ in $M$. musculus and $R$. norvegicus, and G. microti in the one A. flavicollis analyzed, but no evidence for zoonotic G. duodenalis genotypes [15]. In contrast, beavers (Castor spp.) $[9,19]$ and pet chinchillas (Chinchilla lanigera) in particular [35], were found to harbor G. duodenalis assemblage B, indicating a potentially higher risk for zoonotic transmission associated with these host species. In summary, these and the present data highlight the need for local molecular typing analyses to estimate the actual zoonotic risk for Giardia infections emanating from a particular rodent host.

Previous studies from Poland also demonstrated high Giardia spp. prevalences in A. flavicollis, M. glareolus and $M$. arvalis [36, 37] and, in agreement with our results, showed lower parasite cyst loads in samples of Apodemus vs those from Microtus and Myodes. Systematic typing was not performed in these investigations, but four of the Microtus and Myodes samples were exemplarily typed and similar to our study revealed $G$. microti [38]. In this context it should be noted that fecal excretion of Giardia spp. is often not uniform. Therefore, quantitative data on individual time points should be interpreted carefully. However, it is assumed that such effects are averaged out when analyzing aggregated data as that presented here where the means of different groups were compared. Future studies will be necessary to investigate whether host-parasite sequence type relationships as reported here are a consistent finding also in other rodent populations.

Many studies of Giardia spp. in wild rodents used microscopy-based detection of parasite cysts and subsequently attempted to type Giardia by sequencing of PCR amplicons of one or a few marker genes. Often this approach was reported to be inefficient which suggests shortcomings in typing protocols. For example, a recent report on 12 rodent samples (ten $R$. norvegicus, two $M$. musculus) showed that G. duodenalis assemblage G could be typed at all four tested genomic loci ( $b g$, tpi, gdh, SSU rDNA), but G. muris and G. microti-like samples only at the SSU rDNA locus [18]. Primer mismatches that may negatively affect PCR efficacy or low target DNA amounts that may reduce assay sensitivity could be possible reasons. In agreement with these data we also observed lower typing efficiency at $b g$ and gdh loci in comparison to the SSU rDNA locus. We also recognized mismatches of published primers $[4,9,13,28]$ in the G. muris SSU rDNA reference sequences and, therefore, modified primer sets for our semi-nested SSU rDNA PCR assay. Modification resulted in higher positivity rates in particular for G. muris containing samples. In samples of Microtus spp. and Myodes spp., species predominantly infected with $G$. microti, the effect was much less pronounced. Thus to complement IFA data, we consider the semi-nested PCR at the SSU rDNA locus as the most reliable approach to detect the corresponding Giardia species. This approach will even detect non-Giardia species represented by $O$. intestinalis. Alternative PCR methods to discriminate Giardia spp. and G. duodenalis assemblages such as the recently developed tests that amplify $5.8 \mathrm{~S}$ rDNA or internal transcribed spacers (ITS) of ribosomal gene loci [38] may also be suitable but have not been evaluated in this study. PCR and sequencing of $b g$ and $g d h$ loci was more appropriate though for subtyping and description of population structure due to higher sequence heterogeneity. Because only two $b g$ and no $g d h$ sequences were deposited in public databases for G. muris and none of both for G. microti our work significantly enlarges the database which will greatly facilitate future comparative studies.

Our analysis not only confirmed that G. microti is more closely related to the G. duodenalis complex than to G. muris [13] but allowed also to distinguish genetic subgroups and provides evidence that these appear to preferentially infect different hosts. The existence of such subgroups was previously suggested for G. microti based on a very limited number of ribosomal RNA gene sequences [13] and is now supported by other markers through our analysis. This allows the interpretation that possibly various assemblages exist within G. muris and G. microti populations comparable to what has been described for G. duodenalis. Our study further indicates that G. muris also can be divided in sub-types that are preferentially associated with different hosts, in this case illustrated by $A$. agrarius and $A$. flavicollis. Most of the G. muris SSU rDNA sequences newly described were highly divergent from the two published sequences used as references. Both of the latter were derived from samples of non-European rodents [39, 40]. The concept of non-uniformity of $G$. muris is further supported by $b g$ sequences, in spite of the low PCR efficiency at this locus. Further studies are warranted to determine the broader context and significance of phylogenetic relationships (e.g. host range, localities) within G. muris and $G$ microti. This will require improved typing tools. 
We also identified by semi-nested PCR at the SSU rDNA locus three sequences from $O$. intestinalis, a sister lineage from Giardia spp. known to infect wild rodents and together with Giardia spp. form the subfamily Giardiinae [30]. The life-cycle of O. intestinalis is not well characterized and, in particular, information about the morphology and antigenicity of cysts is scarce. All three samples (two Microtus, one Myodes) were also positive in the qPCR analysis and two samples in the IFA analysis. The $b g$ sequence typing was also successful in the latter two samples and revealed G. microti. We therefore consider it likely that co-infections of $G$. microti/O. intestinalis, with a dominance of O. intestinalis, occurred in the respective hosts and that sequence similarity at the SSU rDNA locus of Octomitus spp. to Giardia spp. resulted in detection of the former. This is also corroborated by the high sequence similarity of the primer sequences used in the current study with published reference and primer sequences of the $S S U \mathrm{rDNA}$ sequence of O. intestinalis [30]. Co-infections may also occur with G. microti and G. muris which would impact our interpretations. However, we consider it unlikely that co-infections with different Giardia species are common in our sampled rodent population because, if true, mixed sequences should have been observed frequently.

\section{Conclusions}

In the present study, G. muris was found to be present mainly in Apodemus, and G. microti mainly in Microtus and Myodes hosts. Furthermore, our findings argue for a very low prevalence of zoonotic G. duodenalis assemblages in wild rodents in Germany. Evidence is provided that G. muris and G. microti subdivide into phylogenetically distinct subgroups that each prefers species of a particular rodent host genus (in the case of G. microti) or family (in the case of G. muris). The study also expands the database of sequences relevant for sequence typing of G. muris and G. microti isolates. In future studies this will greatly help to analyze the population structure of these parasites in more detail.

\section{Methods}

\section{Sample collection}

Samples were collected during spring, summer and autumn in 2011 and 2012. Samples collected during field work in four Federal states in Germany were utilized via the German Network "Rodent-borne pathogens" [41]. Rodents were live trapped in "grassland (GL)" and "forest (F)" sites named after the nearby town of the study sites (German Federal State in brackets): Billerbeck (North Rhine-Westphalia, NRW), Weissach (BadenWuerttemberg, BW), Gotha (Thuringia, TH), Jeeser (Mecklenburg-Western Pomerania, MV). A further study site close to Jeeser (GL) was Fuhlendorf-Darß, Saal (MV). In addition, rodents were sampled at six study sites in TH: Kammerforst (Treben) (F), Krahnberg (Gotha) (GL), Leinawald (Altenburg) (F), Pahnaer Holz (Eschefeld) (F), Phönix Ost (Schnauderhainichen) (F), Schaderode (Erfurt) (GL). The latter sites were in reforestation areas and classified according to their "best-match" to GL or F.

Fecal samples (1-2 pellets per animal) were collected from 577 individual animals and preserved in $1 \mathrm{ml} \mathrm{H}_{2} \mathrm{O}$ supplemented with a cocktail of antibiotics in order to inhibit bacterial growth. Samples were shipped at room temperature and stored at $4{ }^{\circ} \mathrm{C}$ until further processing (storage time between 1 and 10 months). Rodent species were identified mainly by field inspection, in some cases molecular typing was performed on fecal samples using cytochrome $b$ as a target gene as described earlier [42].

\section{IFA test}

All samples were examined using the MERIFLUOR ${ }^{\circ}$ Cryptosporidium/Giardia test (Meridian Bioscience, Luckenwalde, Germany) following the manufacturer's protocol and results were qualitatively assigned as being positive or negative for Giardia. In addition, samples were used for a semi-quantitative assignment of cyst numbers in the microscopic sample, which approximates $10 \mu$ of volume:,$+ 1-10$ cysts; ++, 11-50 cysts; +++, > 50 cysts.

\section{DNA isolation}

Genomic DNA was extracted from samples using one of the two commercial kits following the protocols of the manufacturers: QIAamp DNA Stool Mini Kit (Qiagen, Hilden, Germany) or Maxwell 16 FFPE Plus LEV DNA Purification Kit (Promega, Mannheim, Germany). The final elution volume was $70 \mu \mathrm{l}$. Both kits have been tested and performed similarly in subsequent PCR applications. The amount of nucleic acid in samples was photometrically estimated at $\mathrm{OD}_{260}$.

\section{qPCR}

All samples were tested using a TaqMan-based Giardia specific qPCR as earlier described [27, 43] with minor modifications. Briefly, a 62 bp fragment of SSU rDNA was amplified in a total PCR volume of $25 \mu \mathrm{l}[12.5 \mu \mathrm{l}$ Maxima Probe/ROX qPCR Master Mix 2× (ThermoFisher Scientific, Schwerte, Germany); $3 \mu \mathrm{M}$ of each primer (Giardia-80F and Giardia-127R), $1.5 \mu \mathrm{M}$ of double labeled probe (Giardia-105T, 5'FAM, 3'BHQ1) and $1 \mu \mathrm{l}$ of the DNA]. Amplification cycles consisted of $95^{\circ} \mathrm{C}$ for 15 min followed by 40 cycles at $95{ }^{\circ} \mathrm{C}$ for $15 \mathrm{~s}, 60{ }^{\circ} \mathrm{C}$ for $30 \mathrm{~s}$, and $72{ }^{\circ} \mathrm{C}$ for $30 \mathrm{~s}$. Amplification, detection, and data analysis were performed with a Stratagene Mx3000P cycler and according MxPro software (both 
from Agilent Technologies, Waldbronn, Germany). Titration experiments with DNA from G. duodenalis trophozoites (WB6, ATCC 50803) revealed a detection limit (analytical sensitivity) in our technical setting of approximately one trophozoite (equals approximately four genome equivalents) in the PCR. Analytical specificity was calculated as $98 \%$ (calculated from 49 control PCRs of which one was slightly positive with a Ct-value of 38.5). Inhibition was not found to be an issue in the PCR analyses (Only one of 35 tested samples showed a slight inhibition with a delta-Ct value $>2$ in a test set with internal amplification control).

We also used the Ct-value of the GPCR analysis as a proxy for parasite numbers. We assumed that target DNA integrity is similar in the samples' fecal matrix of all three rodent genera and that PCR efficacies were equal, although we were not able to formally test these assay parameters.

\section{Typing PCR and sequencing}

A fragment of the SSU rRNA gene was amplified using a combination of previously described and modified PCR protocols $[4,28]$. A nested PCR protocol was used with the initial primer pair RH11-derivates (equal mix of 5'CAT CCG GTC GAT CCT GCC-3' and 5'-CAT CCG GTT GAT CCT GCC-3') and Gia2150c (5'-CTG CTG CCG TCC TTG GAT GT-3') amplifying a 497 bp product and a secondary primer pair for semi-nested PCR RH11-derivates (equal mix of 5'-CAT CCG GTC GAT CCT GCC-3' and 5'-CAT CCG GTT GAT CCT GCC$3^{\prime}$ ) and RH4-derivates (equal mix of 5'-AGT CGA ACC CTG ATT CTC CGC CAG G-3' and 5'-AGT CAA ACC CTG ATC CTC CGC CAG G-3' and 5'-AGT CGA ACC CTG ATT CTC CGT CAG G-3') amplifying a 293 bp fragment. The PCR consisted of $1 \mu \mathrm{l}$ of DNA for the primary reaction and of $2 \mu \mathrm{l}$ of primary PCR mix for the nested PCR, $200 \mu \mathrm{M}$ dNTPs, $1 \times$ PCR MangoTaq buffer containing $3 \mathrm{mM} \mathrm{MgCl}_{2}$ (Bioline, Luckenwalde, Germany), 2.5 U of MangoTaq polymerase (Bioline), and $200 \mathrm{nM}$ of each primer $(-\mathrm{mix})$ in $25 \mu \mathrm{l}$ in the primary PCR and $50 \mu \mathrm{l}$ reaction in the nested PCR. The reactions were performed for 35 cycles under following conditions: 1st PCR $\left(94{ }^{\circ} \mathrm{C}\right.$ for $45 \mathrm{~s}, 55^{\circ} \mathrm{C}$ for $30 \mathrm{~s}$ and $72{ }^{\circ} \mathrm{C}$ for $45 \mathrm{~s})$ and nested-PCR $\left(94{ }^{\circ} \mathrm{C}\right.$ for $45 \mathrm{~s}, 59{ }^{\circ} \mathrm{C}$ for $30 \mathrm{~s}$ and $72{ }^{\circ} \mathrm{C}$ for $45 \mathrm{~s}$ ). They were run with an initial hot start $\left(95^{\circ} \mathrm{C}\right.$ for $\left.5 \mathrm{~min}\right)$ and a final extension at $72{ }^{\circ} \mathrm{C}$ for 7 min.

Fragments of the $b g$ and $g d h$ genes were amplified according to previously described nested-PCR protocols $[5,7]$. Briefly, PCRs were run in a total volume of $50 \mu \mathrm{l}$ and included 1-2 $\mu \mathrm{l}$ target DNA, $200 \mu \mathrm{M}$ dNTPs, $1 \times$ PCR MangoTaq buffer containing $3 \mathrm{mM} \quad \mathrm{MgCl}_{2}$ (Bioline), $2.5 \mathrm{U}$ of MangoTaq polymerase (Bioline), and $200 \mathrm{nM}$ of each primer. The reactions were performed for 35 cycles using following conditions: 1 st PCR $\left(94{ }^{\circ} \mathrm{C}\right.$ for $45 \mathrm{~s}, 65{ }^{\circ} \mathrm{C}$ (for $b g$ ) and $56{ }^{\circ} \mathrm{C}$ (for $g d h$ ) for $30 \mathrm{~s}$ and $72{ }^{\circ} \mathrm{C}$ for $45 \mathrm{~s}$ ) and nested-PCR $\left(94{ }^{\circ} \mathrm{C}\right.$ for $45 \mathrm{~s}, 65{ }^{\circ} \mathrm{C}$ (for $b g$ ) and $56{ }^{\circ} \mathrm{C}$ (for $g d h$ ) for $30 \mathrm{~s}$ and $72{ }^{\circ} \mathrm{C}$ for $45 \mathrm{~s}$ ). Final extension was done for $7 \mathrm{~min}$ at $72{ }^{\circ} \mathrm{C}$.

All PCR products were analyzed by electrophoresis on $1.2 \%$ agarose gels and visualized after GelGreen (Biotium, Fremont, USA) staining. PCR products of the expected size were purified by using the ExoSAP-IT ${ }^{\circ}$ For PCR Product Cleanup kit (ThermoFisher Scientific). Sequencing reactions were performed in both directions using BigDye 3.1 sequencing reagents (Applied Biosystems) and primer specific annealing temperatures as stated above. The SSU rDNA sequences were compared to selected reference sequences in order to identify Giardia spp. and G. duodenalis assemblages by using built-in applications of Geneious software 9.1. (Biomatters Ltd., Auckland, New Zealand). The following reference sequences were used: G. muris (X65063, AF113895), G. microti (AF006676, AF006676), G. duodenalis assemblage A (M54878), G. duodenalis assemblage B (AF199447), G. duodenalis assemblage C (AF199449), G. duodenalis assemblage D (AF199443), G. duodenalis assemblage E (AF199448), G. duodenalis assemblage F (AF199444), G. duodenalis assemblage G (AF199450), O. intestinalis (DQ366277). For sequence analysis of $b g$ and $g d h$ the following reference sequences were used: $G$ duodenalis assemblage B (AY072725, AY178738), G. duodenalis assemblage A (X85958, M84604), G. muris bg (EF455599), G. ardae gdh (AF069060).

Some of the obtained sequences were analyzed to identify the most similar sequence deposited in the GenBank public database using the built-in Basic Local Alignment Search Tool (BLAST) algorithms (http:// blast.ncbi.nlm.nih.gov/Blast.cgi). Sequence names are deduced from a unique running sample number preceded by an abbreviation of the sequenced genes (e.g. ssu349= SSU rDNA sequence of rodent isolate number 349). Nucleotide sequences generated in this study have been deposited in the GenBank database under accession numbers KY114167-KY114486 (SSU rDNA) and MG676938-MG677109 ( $b g$ and $g d h)$. Accession numbers of each sequence are listed in Additional file 4: Table S2.

\section{Phylogenetic analysis}

Alignments of completely sequenced PCR products (without primer sequences) and respective regions of the reference sequences were produced using MUSCLE [44] integrated subroutine of Geneious version 9.1. (Biomatters Ltd.). Sequences containing polymorphic positions, a wellknown phenomenon in G. duodenalis [45], were included in the analysis when the sequencing data of both strands was available. Bayesian analysis of sequence alignments was performed by using the BEAUti and BEAST software 
packages [46]. PhyML analysis was done with ATGC online tool PhyML 3.0 [47] using the Smart Model Selection tool to select the best substitution model and subsequent PhyML analysis using the best substitution model with Subtree-Pruning-Regrafting (SPR) tree searching and bootstrap performance of 100 [48]. Trees were annotated using the iTOL online tool $[49,50]$. Phylogenetic trait analysis was done using the program BaTS [51].

\section{Statistical analysis}

Data were organized in a spread sheet and subsequently imported into the statistics software package STATA 14. 1 (StataCorp, College Station, USA). Proportions were calculated and analyzed for binomial exact $95 \%$ confidence intervals $(95 \% \mathrm{CI})$. To test for any difference between proportions of groups the Fisher-Freeman-Halton test was used [52]. For comparison between groups, the two-sided Fisher's exact test was used and a multiple correction using the Bonferroni-Holm procedure was applied and adjusted $P$-values are reported [53]. A $P$-value of $\leq 0.05$ was considered statistically significant. Due to the low number of cases we have chosen not to use multivariate analyses using logistic regression models. For qPCR data (Ct values) the median and $\mathrm{min} / \mathrm{max}$ values of groups were calculated and non-parametric Kruskal-Wallis test was performed followed by Dunn's test of multiple comparisons to assess statistical significance using the software package GraphPad Prism 7.03 (a $P$-value of $\leq 0.05$ was considered statistically significant).

\section{Additional files}

Additional file 1: Figure S1. Map of Germany with study sites where wild rodents were captured and sampled. Rodents were captured at 11 study sites that were subdivided into five regions (refer to the color coding) from four German federal states. Please refer to Table 1 for further details on captured animals from each site. (TIFF $780 \mathrm{~kb}$ )

Additional file 2: Table S1. Statistical comparison ( $P$-values) between groups as depicted in Table 1 using Fisher's exact test followed by multiple testing correction (Bonferroni-Holm procedure). (DOCX $17 \mathrm{~kb}$ )

Additional file 3: Figure S2. Bayesian phylogenetic analysis of all SSU rDNA sequence fragments. Unrooted phylogenetic tree comprised of 317 SSU rDNA sequences (277 G. microti, purple clade; 5 G. duodenalis, green clade; 32 G. muris, blue clade and 3 O. intestinalis, red clade). Reference sequences (GenBank accession numbers) of O. intestinalis, G. muris, $G$. duodenalis and G. microti are included (uncoloured sequence names). Posterior probabilities $\geq 0.5$ are illustrated by black dots (proportionally increasing in size). Sequence names are color coded (colored ranges) according to the rodent host. Further color coding (inner to outer layer) was introduced according to locality [1 to 11: 1 ("Billerbeck"), 2 ("Gotha"), 3 ("Krahnberg"), 4 ("Schaderode"), 5 ("Jeeser"), 6 ("Saal"), 7 ("Kammerforst"), 8 ("Leinawald"), 9 ("Pahnaer Holz"), 10 ("Phönix Ost"), 11 ("Weissach"); see also Additional file 1: Figure S1 and Table 1], season (spring, summer, autumn), habitat ( $F=$ "forest", $G L=$ "grassland") and year of sample collection (2011, 2012). Maximum likelihood analysis based on PhyML resulted in a similar tree (not shown). (TIFF $2598 \mathrm{~kb}$ )

Additional file 4: Table S2. Accession numbers of nucleic acid sequences generated in the present study. (XLSX 26 kb)

\section{Abbreviations}

tpi: triosephosphate isomerase; gdh: glutamate dehydrogenase; bg: betagiardin; SSU rDNA: small-subunit ribosomal RNA gene;

IFA: immunofluorescence assay; GPCR: real time-PCR; GL: grassland; F: forest; NRW: North Rhine-Westphalia; BW: Baden-Wuerttemberg; TH: Thuringia; MV: Mecklenburg-Western Pomerania; SNPs: single nucleotide polymorphisms

\section{Acknowledgements}

We would like to acknowledge Petra Gosten-Heinrich from the Robert Koch-Institute for excellent technical assistance, Jörg Thiel (ThüringenForst, Germany) and Kai Thiede (Landesforst MV, Germany) for collection of samples and Stefan Fischer (Friedrich-Loeffler-Institut, Germany) for helpful advice on cytochrome $b$-based rodent species determination. We would also like to thank Dr. Niklas Willrich and Dr. Sebastien Calvignac from Robert Koch-Institute for advice in statistical and phylogenetical analyses.

\section{Funding}

This work was partly supported by the German Federal Ministry for the Environment, Nature Conservation, Building and Nuclear Safety within the UFOPLAN project FKZ 370941401 to Jens Jacob; by the German Federal Ministry of Education and Research (BMBF) through the German Research Platform for Zoonoses (projects FKZ 01 KI1018 and FKZ 01KI1303 to RGU; and project FKZ 01KI1019 to TA) and by the German Research Foundation (DFG) (project number 251133687/ GRK 2046) to CK and TA. YAH was funded by the German Academic Exchange Service (DAAD) and the Ministry of Higher Education in Egypt. Her current affiliation is in Food Animal Health Research Program, Ohio Agricultural Research and Development Center, The Ohio State University, Wooster, Ohio, USA.

\section{Availability of data and materials}

The data supporting the conclusions of this article are included within the article and its additional files. Nucleotide sequences generated in this study have been deposited in the GenBank database under accession numbers KY114167-KY114486 (SSU rDNA) and MG676938MG677109 (bg and $g d h)$.

\section{Authors' contributions}

CK, TA and RGU conceived and designed the experiments. YAH and CK performed the experiments and analyzed the data. JJ and RGU planned and coordinated the field work, including that done by additional partners of the network "Rodent-borne pathogens", and the sample collection and distribution. NGS, SS, UMR, DR and CI performed the field work including the species determination. CK, YAH and TA wrote the paper. All authors read and approved the final manuscript.

\section{Ethics approval}

Animal studies were approved by the responsible local authorities of the German Federal States Baden-Wuerttemberg, North Rhine-Westphalia, Thuringia and Mecklenburg-Western Pomerania (permits 35-9185.82/0261, 8.87-51.05.20.09.210, 22-2684-04-15-107/09, 7221.3-030/09)

Consent for publication

Not applicable.

Competing interests

The authors declare that they have no competing interests.

\section{Publisher's Note}

Springer Nature remains neutral with regard to jurisdictional claims in published maps and institutional affiliations. 


\section{Author details}

'Unit 16 Mycotic and Parasitic Agents and Mycobacteria, Department of Infectious Diseases, Robert Koch-Institute, 13353 Berlin, Germany. ${ }^{2}$ Institute of Novel and Emerging Infectious Diseases, Friedrich-Loeffler-Institut, Federal Research Institute for Animal Health, 17493 Greifswald - Insel Riems, Germany. ${ }^{3}$ Institute for Plant Protection in Horticulture and Forests, Vertebrate Research, Julius Kühn-Institute, Federal Research Centre for Cultivated Plants, 48161 Münster, Germany. ${ }^{4}$ Department Panel Veterinary Public Health, Department of Veterinary Medicine, Freie Universität Berlin, 14163 Berlin, Germany. ${ }^{5}$ Department of Animal Hygiene, Zoonoses and Animal Ethology, Faculty of Veterinary Medicine, Suez Canal University, Ismailia 41511, Egypt. ${ }^{6}$ German Society of Tissue Transplantation, Kruppstraße 98, 45145 Essen, Germany.

\section{Received: 24 November 2017 Accepted: 19 March 2018} Published online: 27 March 2018

\section{References}

1. Ankarklev J, Jerlstrom-Hultqvist J, Ringqvist E, Troell K, Svard SG. Behind the smile. cell biology and disease mechanisms of Giardia species. Nat Rev Microbiol. 2010;8(6):413-22.

2. Klotz C, Aebischer T. The immunological enigma of human giardiasis. Curr Trop Med Rep. 2015;2:119-27.

3. Thompson RC, Monis P. Giardia - from genome to proteome. Adv Parasitol. 2012;78:57-95

4. Hopkins RM, Meloni BP, Groth DM, Wetherall JD, Reynoldson JA, Thompson RC. Ribosomal RNA sequencing reveals differences between the genotypes of Giardia isolates recovered from humans and dogs living in the same locality. J Parasitol. 1997;83:44-51.

5. Lalle M, Pozio E, Capelli G, Bruschi F, Crotti D, Caccio SM. Genetic heterogeneity at the beta-giardin locus among human and animal isolates of Giardia duodenalis and identification of potentially zoonotic subgenotypes. Int J Parasitol. 2005:35:207-13.

6. Lasek-Nesselquist E, Welch DM, Sogin ML. The identification of a new Giardia duodenalis assemblage in marine vertebrates and a preliminary analysis of $G$. duodenalis population biology in marine systems. Int J Parasitol. 2010:40:1063-74.

7. Read CM, Monis PT, Thompson RC. Discrimination of all genotypes of Giardia duodenalis at the glutamate dehydrogenase locus using PCR-RFLP. Infect Genet Evol. 2004;4:125-30.

8. $\quad$ Sprong $H$, Caccio SM, van der Giessen JWB, on behalf of the ZOOPNET network and partners. Identification of zoonotic genotypes of Giardia duodenalis. PLoS Negl Trop Dis. 2009;3:e558.

9. Sulaiman IM, Fayer R, Bern C, Gilman RH, Trout JM, Schantz PM, et al. Triosephosphate isomerase gene characterization and potential zoonotic transmission of Giardia duodenalis. Emerg Infect Dis. 2003:9:1444-52.

10. Monis PT, Caccio SM, Thompson RC. Variation in Giardia. towards a taxonomic revision of the genus. Trends Parasitol. 2009;25:93-100.

11. Monis PT, Andrews RH, Mayrhofer G, Ey PL. Genetic diversity within the morphological species Giardia intestinalis and its relationship to host origin. Infect Genet Evol. 2003;3(1):29-38.

12. Kunstyr I, Schoeneberg U, Friedhoff KT. Host specificity of Giardia muris isolates from mouse and golden hamster. Parasitol Res. 1992;78:621-2.

13. van Keulen $H$, Feely DE, Macechko PT, Jarroll EL, Erlandsen SL. The sequence of Giardia small subunit rRNA shows that voles and muskrats are parasitized by a unique species Giardia microti. J Parasitol. 1998:84:294-300.

14. van Keulen H, Macechko PT, Wade S, Schaaf S, Wallis PM, Erlandsen SL. Presence of human Giardia in domestic, farm and wild animals, and environmental samples suggests a zoonotic potential for giardiasis. Vet Parasitol. 2002;108:97-107.

15. Backhans A, Jacobson M, Hansson I, Lebbad M, Lambertz ST, Gammelgard E, et al. Occurrence of pathogens in wild rodents caught on Swedish pig and chicken farms. Epidemiol Infect. 2013;141:1885-91.

16. Feng Y, Xiao L. Zoonotic potential and molecular epidemiology of Giardia species and giardiasis. Clin Microbiol Rev. 2011;24:110-40

17. Fernandez-Alvarez A, Martin-Alonso A, Abreu-Acosta N, Feliu C, Hugot JP, Valladares $B$, et al. Identification of a novel assemblage $G$ subgenotype and a zoonotic assemblage B in rodent isolates of Giardia duodenalis in the Canary Islands, Spain. Parasitology. 2014;141:206-15.
18. Lebbad M, Mattsson JG, Christensson B, Ljungstrom B, Backhans A, Andersson JO, et al. From mouse to moose. multilocus genotyping of Giardia isolates from various animal species. Vet Parasitol. 2010;168:231-9.

19. Fayer R, Santin M, Trout JM, DeStefano S, Koenen K, Kaur T. Prevalence of Microsporidia, Cryptosporidium spp., and Giardia spp. in beavers (Castor canadensis) in Massachusetts. J Zoo Wildl Med. 2006;37:492-7.

20. Zhao Z, Wang R, Zhao W, Qi M, Zhao J, Zhang L, et al. Genotyping and subtyping of Giardia and Cryptosporidium isolates from commensal rodents in China. Parasitology. 2015;142:800-6.

21. Thompson ARC, Monis PT. Taxonomy of Giardia Species. In: Luján HD, Svärd S, editors. Giardia: a model organism. Wien-New York: Springer; 2011. p. 3-15.

22. WHO. Parasitic Zoonoses. Report of a WHO Expert Committee with the participation of FAO. In: Technical Report Series \#637. Geneva: World Health Organization; 1979.

23. Baker DG. Natural pathogens of laboratory mice, rats, and rabbits and their effects on research. Clin Microbiol Rev. 1998;11:231-66.

24. Meerburg BG, Singleton GR, Kijlstra A. Rodent-borne diseases and their risks for public health. Crit Rev Microbiol. 2009;35:221-70.

25. Padovan D. Infectious diseases of wild rodents. Anacortes, Washington: Corvus Pub. Co.; 2006

26. Perec-Matysiak A, Bunkowska-Gawlik K, Zalesny G, Hildebrand J. Small rodents as reservoirs of Cryptosporidium spp. and Giardia spp. in southwestern Poland. Ann Agric Environ Med. 2015;22:1-5.

27. Verweij JJ, Schinkel J, Laeijendecker D, van Rooyen MAA, van Lieshout L, Polderman AM. Real-time PCR for the detection of Giardia lamblia. Mol Cell Probes. 2003;17:223-5.

28. Appelbee AJ, Frederick LM, Heitman TL, Olson ME. Prevalence and genotyping of Giardia duodenalis from beef calves in Alberta, Canada. Vet Parasitol. 2003;112(4):289-94.

29. Ignatius R, Gahutu JB, Klotz C, Steininger C, Shyirambere C, Lyng M, et al. High prevalence of Giardia duodenalis Assemblage B infection and association with underweight in Rwandan children. PLoS Negl Trop Dis. 2012;6:e1677.

30. Keeling PJ, Brugerolle G. Evidence from SSU rRNA phylogeny that Octomitus is a sister lineage to Giardia. Protist. 2006:157:205-12.

31. Bitto A, Aldras A. Prevalence of Giardia and Cryptosporidium in muskrats in northeastern Pennsylvania and New Jersey. J Environ Health. 2009:71:20-6.

32. Dunlap BG, Thies ML. Giardia in beaver (Castor canadensis) and nutria (Myocastor coypus) from east Texas. J Parasitol. 2002;88:1254-8.

33. Kilonzo C, Li X, Vivas EJ, Jay-Russell MT, Fernandez KL, Atwill ER. Fecal shedding of zoonotic food-borne pathogens by wild rodents in a major agricultural region of the central California coast. Appl Environ Microbiol. 2013;79:6337-44.

34. Karanis P, Opiela K, Al-Arousi M, Seitz HM. A comparison of phase contrast microscopy and an immunofluorescence test for the detection of Giardia spp. in faecal specimens from cattle and wild rodents. Trans R Soc Trop Med Hyg. 1996;90:250-1.

35. Levecke B, Meulemans L, Dalemans T, Casaert S, Claerebout E, Geurden T. Mixed Giardia duodenalis assemblage A, B, C and E infections in pet chinchillas (Chinchilla lanigera) in Flanders (Belgium). Vet Parasitol. 2011;177:166-70.

36. Bajer A. Between-year variation and spatial dynamics of Cryptosporidium spp. and Giardia spp. infections in naturally infected rodent populations. Parasitology. 2008;135:1629-49.

37. Bajer A, Bednarska M, Pawelczyk A, Behnke JM, Gilbert FS, Sinski E. Prevalence and abundance of Cryptosporidium parvum and Giardia spp. in wild rural rodents from the Mazury Lake District region of Poland. Parasitology. 2002;125:21-34.

38. Caccio SM, Beck R, Almeida A, Bajer A, Pozio E. Identification of Giardia species and Giardia duodenalis assemblages by sequence analysis of the 5.8S rDNA gene and internal transcribed spacers. Parasitology. 2010;137:919-25.

39. Mayrhofer G, Andrews RH, Ey PL, Chilton NB. Division of Giardia isolates from humans into two genetically distinct assemblages by electrophoretic analysis of enzymes encoded at 27 loci and comparison with Giardia muris. Parasitology. 1995;111:11-7.

40. van Keulen H, Gutell RR, Campbell SR, Erlandsen SL, Jarroll EL. The nucleotide sequence of the entire ribosomal DNA operon and the structure of the large subunit rRNA of Giardia muris. J Mol Evol. 1992:35:318-28.

41. Ulrich RG, Heckel G, Pelz HJ, Wieler LH, Nordhoff M, Dobler G, et al. Rodents and rodent associated disease vectors: the network of "rodent carrying pathogens" introduces itself. Bundesgesundheitsbl. 2009;52:352-69. 
42. Schlegel M, Ali HS, Stieger N, Groschup MH, Wolf R, Ulrich RG. Molecular identification of small mammal species using novel cytochrome B genederived degenerated primers. Biochem Genet. 2012;50:440-7.

43. Helmy YA, Klotz C, Wilking H, Krucken J, Nockler K, Von SamsonHimmelstjerna G, et al. Epidemiology of Giardia duodenalis infection in ruminant livestock and children in the Ismailia province of Egypt: insights by genetic characterization. Parasit Vectors. 2014;7:321.

44. Edgar RC. MUSCLE. multiple sequence alignment with high accuracy and high throughput. Nucleic Acids Res. 2004;32:1792-7.

45. Caccio SM, Ryan U. Molecular epidemiology of giardiasis. Mol Biochem Parasitol. 2008;160:75-80

46. Drummond AJ, Suchard MA, Xie D, Rambaut A. Bayesian phylogenetics with BEAUti and the BEAST 1.7. Mol Biol Evol. 2012;29:969-1973.

47. Guindon S, Dufayard JF, Lefort V, Anisimova M, Hordijk W, Gascuel O. New algorithms and methods to estimate maximum-likelihood phylogenies. assessing the performance of PhyML 3.0. Syst Biol. 2010;59:307-21.

48. PhyML 3.0. new algorithms, methods and utilities. https://www.atgcmontpellier.fr/phyml/. Accessed 14. Nov 2017.

49. Interactive Tree Of Life. https://itol.embl.de/. Accessed 14 Nov 2017.

50. Letunic I, Bork P. Interactive tree of life (iTOL) v3. an online tool for the display and annotation of phylogenetic and other trees. Nucleic Acids Res. 2016:44:242-5.

51. Parker J, Rambaut A, Pybus OG. Correlating viral phenotypes with phylogeny. accounting for phylogenetic uncertainty. Infect Genet Evol. 2008;8:239-46.

52. Freeman $\mathrm{GH}$, Halton JH. Note on an exact treatment of contingency, goodness of fit and other problems of significance. Biometrika. 1951;38:141-9.

53. Holm S. A simple sequentially rejective multiple test procedure. Scand J Statist. 1979;6:65-70

\section{Submit your next manuscript to BioMed Central and we will help you at every step:}

- We accept pre-submission inquiries

- Our selector tool helps you to find the most relevant journal

- We provide round the clock customer support

- Convenient online submission

- Thorough peer review

- Inclusion in PubMed and all major indexing services

- Maximum visibility for your research

Submit your manuscript at www.biomedcentral.com/submit 